\title{
Comparison of the frailty phenotype and the Tilburg Frailty Indicator regarding the prediction of quality of life in a two-year follow-up
}

\author{
T. Coelho ${ }^{1}$ \\ C. Paúl $\left.\right|^{2}$ \\ L. Fernandes ${ }^{3}$ \\ 1 School of Allied Health Technologies, Polytechnic Institute of Porto, Department of \\ Occupational Therapy, Vila Nova de Gaia, Portugal \\ 2 Institute of Biomedical Sciences Abel Salazar, University of Porto, UNIFAI/CINTESIS, Porto, \\ Portugal \\ 3 Faculty of Medicine, University of Porto, UNIFAI/CINTESIS, Porto, Portugal
}

\section{Introduction}

Frail individuals are highly vulnerable to minor stressful events, presenting a higher risk for adverse health outcomes (e.g. falls, disability, hospitalization), which can lead to a decline in quality of life (QoL). In this context, an early screening of elderly frailty is of crucial importance.

\section{Objective}

To compare how the Frailty Phenotype (FP) and the Tilburg Frailty Indicator (TFI) predict QoL in a two-year follow-up.

\section{Methods}

A longitudinal study was designed recruiting 110 community-dwelling elderly ( $\geq 65$ years). The presence of frailty was assessed at baseline (FP $\geq 3$ and TFI $\geq 6$ ), whereas QoL was measured two years later with two different scales: the WHOQOL-OLD and the EUROHIS-QOL-8. Hierarchical regressions were conducted.

\section{Results}

The mean age of the participants at baseline was $77.7 \pm 6.9$ years, and most were women (75.5\%). According to FP, $33.6 \%$ of the participants were classified as frail, while the TFI detected frailty in $50 \%$ of the elderly. After adjusting for age and gender, the TFI significantly predicted QoL $\quad$ (WHOQOL-OLD: $\quad \beta=-18.9, t(106)=-6.97, P<0.001 ; \quad$ EUROHIS-QOL-8: $\beta=-6.1, t(106)=-6.71, P<0.001)$, whereas the effect of the FP on the outcome measures was non-significant.

\section{Conclusions}

Frailty at baseline was associated with a lower QoL at follow-up. A multidimensional frailty operationalization (TFI) showed a stronger predictive validity than an exclusively physical one (FP). The option of which frailty measure to use in a clinical setting should take into account its ability to predict specific adverse outcomes, conducing to targeted and effective interventions. 